\title{
The Photon Veto System for the NA62 Rare Kaon Decay Experiment
}

\section{A. Antonelli ${ }^{a}$, F. Ambrosino ${ }^{b}$, F. Costantini ${ }^{*}$, G. D'agostini ${ }^{d}$, B. Dulach ${ }^{a}, \mathbf{R}$.} Fantechi $^{c}$, G. Lamanna ${ }^{e}$, E. Leonardi ${ }^{d}$, I. Mannelli ${ }^{e}$, P. Massarotti ${ }^{b}$, M. Moulson ${ }^{a}$, M. Napolitano ${ }^{b}$, V. Palladino ${ }^{b}$, F. Raffaelli ${ }^{c}$, M. Raggi $^{a}$, A. Romano ${ }^{b}$, G. Saracino ${ }^{b}$, M. Serra ${ }^{d}$, T. Spadaro ${ }^{a}, \mathbf{P}$. Valente ${ }^{d}$ and S. Venditti ${ }^{\dagger c}$

a, INFN Frascati National Laboratories

Via E. Fermi, 40 - 00044 Frascati (RM)

${ }^{b}$, University of Naples \& INFN

Via Cintia - 80126 Napoli

${ }^{c}$, University of Pisa \& INFN

Largo B. Pontecorvo, 3 - 56127 Pisa

$d$, University of Rome "La Sapienza" \& INFN

P.le Aldo Moro, 2 - 00165 Roma

e Scuola Normale Superiore \& INFN

Piazza dei Cavalieri, 7 - 56126 Pisa

\begin{abstract}
Three possible solutions for a system of large-angle vetoes (LAV) have been analyzed and tested in order to choose the best option for the NA62 experiment, expected to take data in 2011-12 at CERN. The rejection power reached by the veto system, whose goal is mainly to veto photons from the $\mathrm{K}^{+} \rightarrow \pi^{+} \pi^{0}$ and $\mathrm{K}^{+} \rightarrow \pi^{+} \pi^{0} \pi^{0}$ decays, is a key-point to collect $\sim 100$ events of the $\mathrm{K}^{+} \rightarrow \pi^{+} v \bar{v}$ decay $\left(\mathrm{BR} \sim 10^{-10}\right.$ ) with a total background (BG) of the order of $10 \%$, thus providing a very stringent test of the Standard Model $(\mathrm{SM})$ and a measurement of the $\mathrm{V}_{t d}$ element of the Cabibbo-Kobayashi-Maskawa (CKM) matrix. The details of this study and the motivations of the choice among tested options will be reviewed in this paper.
\end{abstract}

2009 KAON International Conference KAON09,

June 09 - 12, 2009

Tsukuba, Japan

\footnotetext{
*Speaker.

† corresponding author. E-mail: stefano.venditti@pi.infn.it
} 


\section{The $\mathbf{K}^{+} \rightarrow \pi^{+} v \bar{v}$ decay}

The attention of many particle physics theoreticians has been recently attracted by some "golden" decay channels, for which the theoretical prediction on the branching ratio (BR) is particularly precise. A comparison of these predictions with experimental measurements performed with similar precision would represent a stringent test of the Standard Model (SM), either confirming its strength or signalling the presence of new physics which would require an extension of the model itself. One of these channels is the $\mathrm{K}^{+} \rightarrow \pi^{+} v \bar{v}$ decay, whose Feynman diagrams are shown in fig. 1 .

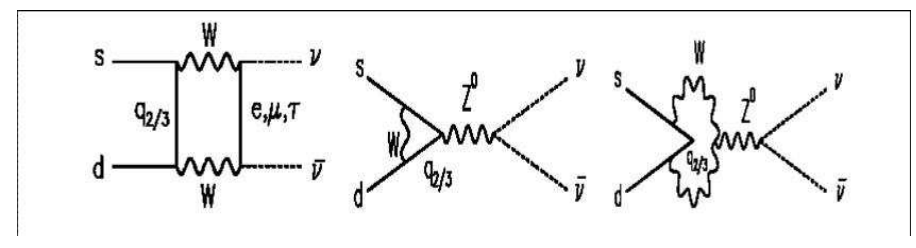

Figure 1: Feynman diagrams for the $\mathrm{K}^{+} \rightarrow \pi^{+} v \bar{v}$ decay.

The precise prediction on the $\mathrm{K}^{+} \rightarrow \pi^{+} v \bar{v}$ BR value stems from the possibility of relating the FCNC hadronic matrix element of the decay to kaon semileptonic decays (especially to $\mathrm{K}^{+} \rightarrow$ $\left.e^{+} \pi^{0} v\right)$ by isospin symmetry. The BR expression is [1]:

$$
B R\left(K^{+} \rightarrow \pi^{+} v \bar{v}\right)=k_{+}\left(1+\Delta_{E M}\right) \cdot\left[\left(\frac{\operatorname{Im} \lambda_{t}}{\lambda^{5}} X\left(x_{t}\right)\right)^{2}+\left(\frac{\operatorname{Re} \lambda_{c}}{\lambda} P_{c}(X)+\frac{\operatorname{Re} \lambda_{t}}{\lambda^{5}} X\left(x_{t}\right)\right)^{2}\right]
$$

where $\lambda$ is the Wolfenstein parameter, $\mathrm{k}_{+}$is proportional to the hadronic part, $\lambda_{i}=\mathrm{V}_{i s}^{*} \mathrm{~V}_{i d}$ are products of CKM matrix elements and $\Delta_{E M}$ represents a negligible electromagnetic correction. The dependence of the $\mathrm{X}$ factors by $\mathrm{x}_{i}=\mathrm{M}_{q}^{2} / M_{W}^{2}$, X representing the summed contributions of diagrams with quarks up, charm and top to the total amplitude, is such that the contribution of the up quark can be safely neglected.

The total error on the theoretical prediction depends on parametric uncertainty $(\sim 70 \%$, mostly due to CKM matrix elements) and on theory $(\sim 30 \%)$. For what concerns the latter, the uncertainty on $\mathrm{X}\left(\mathrm{x}_{t}\right)$ is dominated by the top mass error $\left(\mathrm{m}_{t}=161.0 \pm 1.7 \mathrm{GeV}\right)$ and will become negligible once LHC data will allow to decrease such an error well below the $1 \mathrm{GeV}$ level. The uncertainty on $\mathrm{P}_{c}(X)$ is dominated by the precision on long-distance contributions and on the c quark mass measurement $\left(\mathrm{m}_{c}=1286 \pm 13 \mathrm{MeV}\right)$ and represents the largest contribution to the theoretical error, although its size is smaller compared to the top contribution. The present theoretical estimation of the $\mathrm{K}^{+} \rightarrow \pi^{+} v \bar{v} \mathrm{BR}$ is $(0.85 \pm 0.07) \cdot 10^{-10}$ [2]; the precision is foreseen to reach $\simeq 5 \%$ in the next years.

On the experimental side, 7 candidate events were detected by the E787/E949 collaborations at FNAL [3], yielding a BR measurement of $\left(1.73 \pm_{-1.05}^{+1.15}\right) \cdot 10^{-10}$.

\section{The NA62 experiment at CERN}

A sketch of the NA62 experiment [4] is shown in fig. 2. 


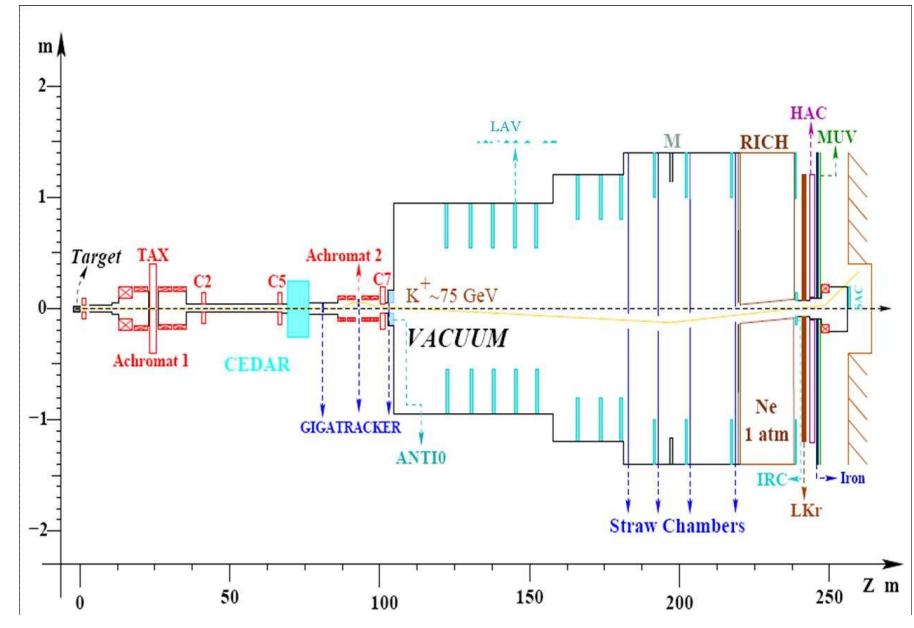

Figure 2: Sketch of the NA62 experiment.

The main goal of the NA62 experiment, based in the North Area (hence NA) at CERN, is to collect $\sim 100 \mathrm{~K}^{+} \rightarrow \pi^{+} v \bar{v}$ candidate events in 2 years of data-taking, with an expected BG of $\sim 10 \%$. In order to reach this goal, the combined effect of the detectors used must allow to tag other kaon decays up to the required rejection level, which depends on each channel's BR. $\mathrm{K}^{+}$ are produced by impinging the $400 \mathrm{GeV} / \mathrm{c}$ SPS protons on a beryllium target and selected in an energy range of $75 \pm 1 \% \mathrm{GeV} / \mathrm{c}$ through an achromat system; subsequently they decay along the beam pipe. The detectors can be subdivided according to the way in which the event tagging is performed, by :

- Kinematical rejection, where the squared missing mass of the pion (defined as $\mathrm{MM}_{\pi}^{2}=\mid \mathrm{K}$ $\left.\pi^{+}\right|^{2}$ ) is reconstructed with high precision and used to tag $\mathrm{K}^{+} \rightarrow \pi^{+} v \bar{v}$ decay. As a function of kaon and pion momenta and directions, $\mathrm{MM}_{\pi}^{2}$ can be written as:

$$
M M_{\pi}^{2}=m_{K}^{2}\left(1-\frac{\left|P_{\pi}\right|}{\left|P_{K}\right|}\right)+m_{\pi}^{2}\left(1-\frac{\left|P_{K}\right|}{\left|P_{\pi}\right|}\right)-\left|P_{K}\right|\left|P_{\pi}\right| \theta_{K \pi}
$$

The detectors used for the $\mathrm{MM}_{\pi}^{2}$ measurement will be the Gigatracker, aiming at measuring the incoming kaon's momentum and direction with high precision and at standing a particle rate up to the $\mathrm{GHz}$, and the straw chambers, measuring the momentum and direction of charged particles coming from kaon decays. Two $\mathrm{MM}_{\pi}^{2}$ regions (I and II, see fig. 3 left) have been defined for the $\mathrm{K}^{+} \rightarrow \pi^{+} v \bar{v}$ BR measurement, as in the remaining $\mathrm{MM}_{\pi}^{2}$ spectrum the high BG from other channels does not allow to obtain the required level of event rejection;

- PID rejection, based on Cherenkov effect. The latter is used by CEDAR to tag kaons in the incoming beam and distinguish them from other particles (mainly protons and pions) and by the RICH (Ring Imaging CHerenkov) to distinguish pions from muons by kaons' decays;

- Vetoes, aiming at detecting particles produced in BG events: the most important are the Large Angle Vetoes (LAV), an array of 12 annular veto stations placed along the decay line, 
the Liquid Krypton calorimeter ( $\mathrm{LKr}$ ), used to detect forward electrons and photons, and the muon veto, detecting muons.

In fig. 3 the $\mathrm{MM}_{\pi}^{2}$ distributions for constrained ( $\simeq 92 \%$ of total $\mathrm{K}^{+}$decays) and unconstrained BG (i.e. that cannot be reduced using $\mathrm{MM}_{\pi}^{2}, \simeq 8 \%$ ) are compared to that of $\mathrm{K}^{+} \rightarrow \pi^{+} v \bar{v}$ decay (no relative normalization). Adequate rejection on a single channel is often obtained through a combination of the mentioned techniques: as an example in the $\mathrm{K}^{+} \rightarrow \pi^{+} \pi^{0}$ rejection is performed combining $\mathrm{MM}_{\pi}^{2}$ reconstruction and photon vetoing.
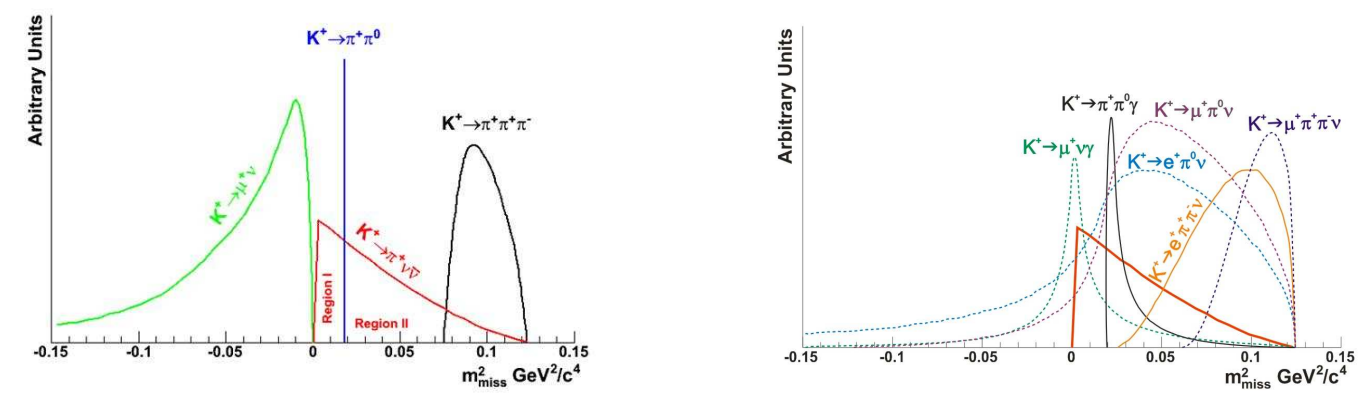

Figure 3: Constrained (left) and unconstrained (right) $\mathrm{MM}_{\pi}^{2} \mathrm{BG}$ distribution.

\section{Large angle vetoes}

The large angle veto system represents a key-detector in the experiment: an accurate vetoing of high transverse momentum particles (mainly photons from $\pi^{0}$ decay) along the beam pipe is mandatory to reach the BG rejection level required for the experiment. Simulations have shown that the required detection inefficiency must be at the level of $10^{-3}-10^{-4}$ for particles ranging from few hundred MeV's to some GeV's. As a consequence, particular care has been taken by the collaboration in choosing the best possible experimental solution. During the R\&D phase, three options were tested:

- A lead+scintillating fiber solution, based on the KLOE calorimeter; to this purpose a horseshoeshaped prototype was built and tested in Frascati in 2007, whose shape and size was that of the envisaged veto stations. The thickness was $16.8 \mathrm{~cm}$ (all fibers, $13 \mathrm{X}_{0}$ ) $+8.2 \mathrm{~cm}$ (fibers+lead,10 $\mathrm{X}_{0}$ ), for a total of 18 readout cells, $4.2 \times 4.2 \mathrm{~cm}^{2}$ cross section;

- A lead+scintillating tile solution, based on the CKM experiment vetoes [5]. To test this solution a prototype (1/16 of a ring) was shipped to Frascati from FNAL in 2007 in order to be tested. The particle crosses $16 \mathrm{X}_{0}$ of material, made of scintillating tiles ( $5 \mathrm{~mm}$ each) and lead foils (1mm each). Fibers inserted in the tiles drive light directly to the photomultiplier;

- A lead glass crystal solution. The idea was to reuse the $\sim 10^{4}$ crystals from the OPAL experiment $\left(\mathrm{X}_{0}=1.5 \mathrm{~cm}, \rho=5.6 \mathrm{~g} / \mathrm{cm}^{3}\right.$, light produced by Cherenkov effect), and organize them in a suitable geometry in order to match the required efficiency. Tests on arrays of crystals were performed in Frascati and Naples in 2007 in order to assess their performances. 


\begin{tabular}{|c|c|c|c|}
\hline Station & Rings & Crys/ring & Radius $(\mathrm{cm})$ \\
\hline I to V & 5 & 32 & 96 \\
\hline VI to VIII & 5 & 48 & 120 \\
\hline IX to XI & 4 & 60 & 140 \\
\hline XII & 4 & 64 & 150 \\
\hline
\end{tabular}

Table 1: Characteristics of the 12 veto stations.

The three prototypes were tested at the Beam Test Facility in the Frascati National Laboratories, with a tagged electron beam of variable energy. The result of the tests performed are summarized in fig. 4: inefficiency, defined as the fraction of events crossing a certain fiducial region of the prototype and producing a signal lower than $50 \mathrm{MeV}$ (equal to $\sim 10$ photoelectrons), was measured for all the three prototypes as a function of the incoming particles' energies. The outcome of the tests was that all the three tested solutions matched the requirements of the experiment: the lead glass option was then chosen, as it represented the cheapest and least time-consuming solution.

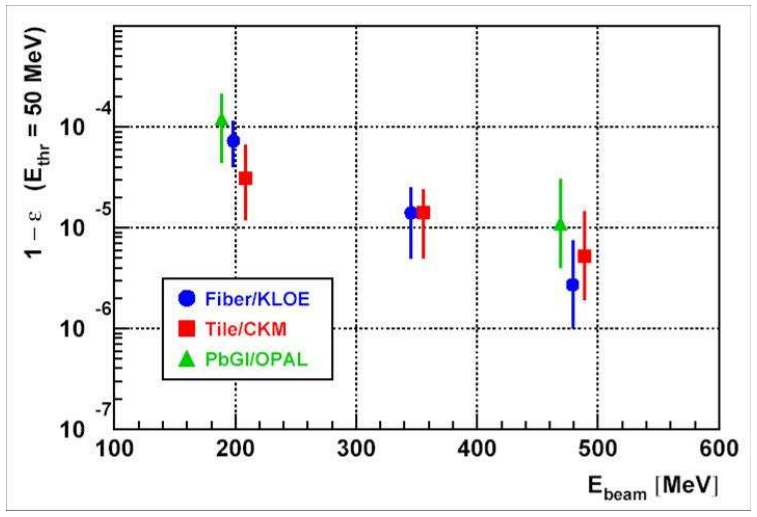

Figure 4: Electron inefficiency of the three tested solutions as a function of the particle's energy.

\section{Veto layout and 2008 tests}

Once the use of the lead-glass crystals was settled, the geometry of the veto station was studied in order to optimize the veto efficiency. It was decided to realize 12 veto station of increasing size along the beam line, each one made of 5 or 4 staggered crystal rings. In table 4 the characteristics of the 12 stations are shown.

The crystals, whose axis points at the beam line, are organized in units of 4 and share a common structure (see fig. 5) which allows their placement inside the vessel after passing cosmic ray calibration and pulser tests. Accurate tests were performed at CERN in September 2008 on a prototype of 1/8 the size of a type I station (see fig. 6), which was put on the NA62 beam line and irradiated with photons from kaon decays and muons. 


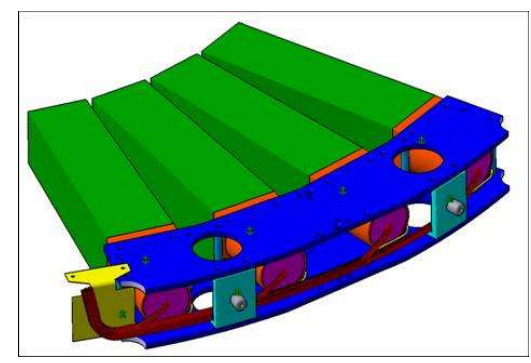

Figure 5: Structure mounting 4 crystals.

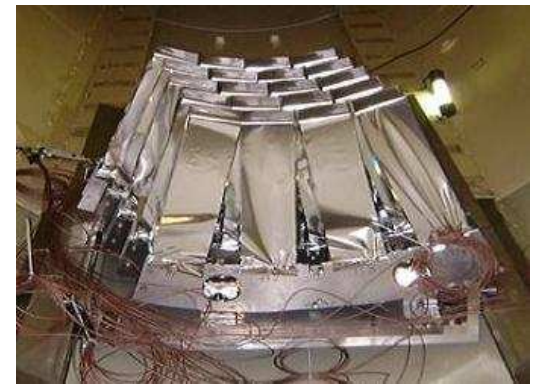

Figure 6: The prototype used in the 2008 test.

\section{First module construction and future plans}

The first of the 12 stations (see fig. 7) was assembled in Frascati in May-June 2009 and shipped to CERN in July, while in August it has been installed on the NA62 beam line in view of further on-beam tests scheduled for the last quarter of the year. The construction of the remaining stations will begin in 2010 in order to fulfill the experiment's schedule.

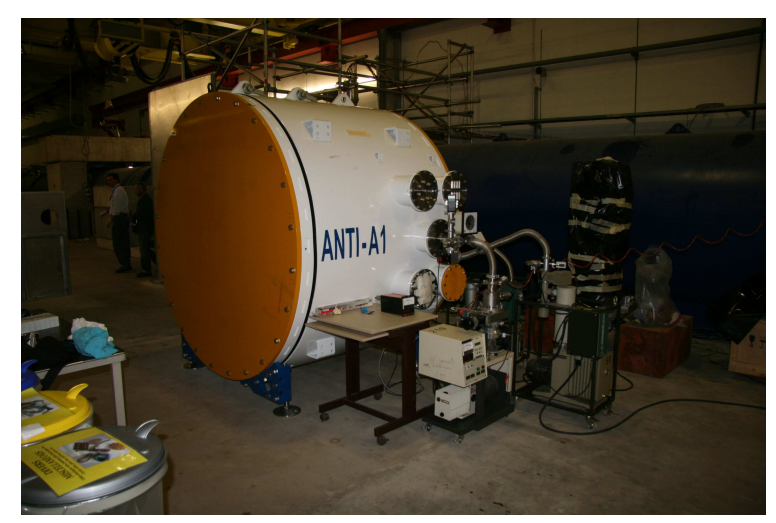

Figure 7: The first station of the veto system.

\section{References}

[1] A. Buras, F. Schwab and S. Uhlig, Rev. Mod. Phys. 80, 965-1007 (2008);

[2] J. Brod and M. Gorbahn, Phys. Rev. D78, 034006 (2008);

[3] E787/E749 collaboration, Phys. Rev. Lett. 101,191082 (2008);

[4] Anelli et al., SPSC report, CERN 013 (2005);

[5] E. Ramberg, P. Cooper and R. Tschirart, IEEE Trans. Nuc. Sci. 51, 2201 (2004). 